Ann. Sc. forest., 1978, 35 (1), 33-53.

\title{
Nutrition et production de l'Epicea commun adulte sur sols hydromorphes en Lorraine : liaisons avec les caractéristiques stationnelles
}

\author{
G. LEVY \\ avec la collaboration technique de M. GUILLEMETTE et Y. LEFEVRE \\ Centre National de Recherches forestières. Station de Recherches sur les Sols Forestiers \\ ef la Fertilisation INRA. Champenoux, 54280 Seichamps
}

\section{Résumé}

L'Epicéa commun est très utilisé dans l'Est de la France en reboisement des sols hydromorphes, ce qui a motivé cette étude station-nutrition-production, réalisée en Lorraine dans une gamme très variée de stations.

La production (qui varie de 4 à $22 \mathrm{~m}^{3} / \mathrm{ha} / \mathrm{an}$ ) est très liée à la teneur du sol en argile, à la stabilité de sa structure, et à l'indice de drainage (variable définie par l'A.) ; le coefficient de corrélation multiple est de 0,86 , du moins pour l'ensemble des stations ne présentant pas en profondeur en plus d'une nappe temporaire relativement superficielle de nappe " permanente " (au sens large) ; cette dernière éventualité permet de gagner en moyenne une classe de production.

Les analyses foliaires ont surtout mis en évidence un déficit azoté apparaissant principalement au cours des années humides et dans les placettes à faible indice de drainage.

Nappe superficielle, mauvaise stabilité structurale du sol, et forte proportion d'argile contribuent à réduire le volume de sol prospecté par les racines, donc affectent l'alimentation en eau de l'Epicéa ; déficit hydrique et carence azotée sont ainsi sans doute directement à l'origine de la baisse de production constatée.

Des critères simples peuvent donner au praticien par examen du profil et observation de la nappe une certaine idée de la production potentielle de l'Epicéa dans les diverses stations hydromorphes où il envisage d'introduire éventuellement cette essence.

\section{Introduction}

Les sols forestiers à nappe temporaire sont très répandus dans le Nord Est de la France.

On y introduit souvent de l'Epicéa commun, quand la production des peuplements feuillus est considérée comme insuffisante ou de mauvaise qualité et parfois lorsque leur régénération naturelle est très difficile et a échoué. La grande variété des caractéristiques écologiques qu'on y rencontre motivait une étude des relations stationnutrition-production pour cette essence. Une première phase de l'étude ( $N$. Decourt et G. Levy, 1974) a montré que 90 p. 100 des peuplements retenus se situaient dans les trois meilleures classes de productivité ; mais la gamme étendue des productions (de 4 à $22 \mathrm{~m}^{3} / \mathrm{ha} / \mathrm{an}$ ) renforçait l'intérêt de notre entreprise. 
Des problèmes spécifiques sont apparus, par rapport aux travaux du même type : le facteur écologique plus spécialement étudié, qui est la nappe, varie en effet en permanence, tant en ce qui concerne ses fluctuations que ses propriétés, tel le potentiel d'oxydoréduction.

\section{1. - Matériel et méthodes}

\section{1. - Les stations}

La méthode générale d'étude des liaisons stations-caractéristiques des peuplements (Garbaye et al., 1970) a servi de base à ce travail. Quatre-vingt-dix-sept microplacettes ont été installées (quelques-unes n'ont pu être utilisées complètement, le peuplement ayant été exploité depuis) ; l'âge était compris entre 36 et 69 ans.

Ces placettes sont situées en Lorraine, sous un climat relativement homogène ; la pluviométrie moyenne s'échelonne tout de même de 700 à $1000 \mathrm{~mm}$ par an. Elles représentent pratiquement tous les types de stations à hydromorphie temporaire de cette région. Tous les genres de marmorisation apparaissent, depuis des taches peu prononcées et profondes jusqu'à un bariolage très accentué remontant à la base de I'horizon $A_{1}$. De même, le «plancher » de la nappe est formé soit d'une couche géologique argileuse (pseudogley primaire), soit de l'horizon $B_{g}$ d'un sol formé sur limons (pseudogley secondaire) et il peut apparaître à des profondeurs très variables : entre plus de $1 \mathrm{~m}$ et la surface du sol. Les sols varient ainsi du sol brun marmorisé au pélosol-pseudogley et au pélosol. Ce dernier n'est pas à proprement parler un sol hydromorphe, mais il s'en rapproche beaucoup par ses propriétés vis-à-vis du peuplement : il y a saturation de la porosité par l'eau en période humide ; de plus la macrostructure souvent très stable et l'épaisseur de son horizon $A_{1}$ (mull « pélosolique », intermédiaire dans une certaine mesure entre mull calcique et mull eutrophe) permettent la présence d'eau libre formant en quelque sorte une nappe perchée.

Signalons enfin que certains substrats se distinguent de ceux de la grande majorité des placeties : alluvions anciennes, et même une tourbe drainée.

\section{2. - Les mesures}

La hauteur dominante à 50 ans, indice de productivité retenu, a été obtenue par reconstitution approximative de la courbe de croissance d'arbres dominants grâce à des sondages à la tarière de Pressler à différentes hauteurs sur leur tronc ( $N$. Decourt et G. Levy, 1974).

Afin d'être en mesure de comparer plus valablement les propriétés (surtout physiques) des différents sols, nous avons effectué les prélèvements à des profondeurs « standard» : 0-8 (prélèvement 1), 12-20 (prélèvement 2), 24-32 (prélèvement 3), 40-48 (prélèvement 4), 56-64 (prélèvement 5), et 80-88 cm (prélèvement 6), en décalant légèrement ces limites quand c'était nécessaire afin d'éviter un mélange d'horizons. Cependant, seuls les sols de vingt placettes, représentant toute la gamme des stations, ont été complètement analysés dans un premier temps. Après étude de corrélations avec la productivité, nous n'avons effectué sur les autres sols que les analyses à priori intéressantes. 
II était évidemment impossible de suivre les fluctuations des nappes dans un aussi grand nombre de stations; d'ailleurs l'objectif précis de cette étude ne nécessitait que l'obtention, pour chaque placette, d'un indice représentatif du caractère plus ou moins superficiel et durable de la nappe. Nous avons ainsi appelé «indice de drainage » le niveau d'apparition de la nappe mesuré en hiver un jour (le même pour toutes les placettes) où les nappes sont dans l'ensemble assez hautes. Nous avons en effet constaté (étude publiable prochainement) que pour des textures voisines, le classement des stations en fonction de la profondeur de la nappe reste à peu près le même, à conditions climatiques identiques, quelle que soit la date de mesure. Pour un ensemble de stations à textures variées, ce résultat ne reste valable que pendant l'hiver : en automne et au printemps, pour un même niveau hivernal, la nappe est d'autant plus superficielle qu'une texture lourde apparaît moins profondément.

Nous avons ainsi mesuré le niveau des nappes à plusieurs reprises chaque hiver entre l'automne 1970 et le printemps 1974, dans les quatre piézomètres installés dans chacune des placettes. En effet, il s'agissait de sélectionner une date à laquelle les nappes étaient particulièrement superficielles, mais il fallait aussi qu'il ne se soit pas produit d'anomalies locales dans les précipitations (pluies importantes sur une partie des placettes seulement) au cours des jours précédents.

Malheureusement, ces hivers-là ne furent pas très humides, et à la date finalement choisie (le 16.2.1971), la nappe était absente dans trente-sept placettes (l'indice 50 leur a été affecté par convention). Le 11.2.1974, avec seulement dix placettes sans nappe, aurait aussi pu être retenu, malgré certaines hétérogénéités climatiques (on a d'ailleurs pu vérifier que l'interprétation globale était pratiquement la même pour ces deux dates).

Il est apparu qu'il existait dans certaines placettes une saturation en eau subsistant même (en général vers $-2 \mathrm{~m}$ ) pendant les périodes les plus sèches de l'été. A cette profondeur, la texture est souvent très argileuse ; il n'y a alors probablement pas de pores non capillaires et il ne s'agit pas vraiment d'une nappe libre ; nous utiliserons le terme de «zone saturée d'été ».

Les analyses foliaires ont été faites sur des prélèvements effectués sur cinq arbres dominants dans chaque placette au fusil ou, quand c'était nécessaire, par cueillette, à l'automne de 1969 et 1970 . Ces deux années ont eu un climat fort différent; ainsi à Amance, la pluviosité au cours des six premiers mois fut moyenne en 1969 (429 mm), forte en 1970 (604 mm), alors que les mois de juillet à octobre furent beaucoup plus secs en 1969 qu'en 1970 (respectivement 126 et $256 \mathrm{~mm}$ ) ( ${ }^{1}$ ).

A plusieurs reprises, dans des conditions climatiques variées, nous avons effectué, dans quatorze placettes bien représentatives de l'ensemble, des mesures du potentiel d'oxydo-réduction à l'aide d'un appareil portatif muni d'électrodes au platine et au calomel. Ces relevés furent faits à la fois dans la nappe et dans l'horizon $A_{1}$ lorsqu'il était ennoyé ou au moins saturé.

Nous avons réalisé, par ailleurs, une étude rapide de la mycorhization des racines localisées en $A_{1}$ dans vingt-deux placettes-témoins : estimation à la loupe de la densité de mycorhizes totales et de mycorhizes actives (d'après leur teinte et leur aspect général).

( $\left.{ }^{1}\right)$ Ces chiffres nous ont été aimablement communiqués par Monsieur Aussenac. 
Les principales caractéristiques des placettes sont inentionnées sur un tableau 1, à la disposition des lecteurs qui en feront la demande à l'auteur.

\section{2. - Liaisons station-production}

\section{1. - Etude par des méthodes statistiques}

\subsection{Résultats.}

Les résultats les plus intéressants sont constitués par les coefficients de corrélation totale et par les régressions progressives.

Cette étude a comporté deux stades successifs.

Dans le premier, seules vingt placettes ont été retenues (on y a effectué des analyses complètes du sol). Elles avaient été choisies de façon à représenter l'ensemble de la variation à la fois des productions et des types de sol. La hauteur dominante à 50 ans (HD 50) était la variable à " expliquer », les variables explicatives élant constituées de l'ensemble des propriétés des stations : propriétés physiques, chimiques du sol, variables topographiques, pluviométrie, indice de drainage. L'objectif de cette opération était de dégager les variables liées effectivement à la production afin d'alléger l'étude des autres placettes, tant sur le plan analyses qu'interprétation statistique.

L'ensemble des résultats statistiques a ainsi permis de ne retenir que les variables suivantes : teneurs en $\mathrm{Mg}$ et $\mathrm{K}$ et pourcentage d'argile des prélèvements 2,3 et 4 , teneur en matière organique et indice ls des prélèvements 2 et 3 , indice de drainage, pluviométrie.

Le second stade intéresse l'ensemble des placettes. Les coefficients de corrélation totale les plus élevés entre la production et les variables retenues concernent : Mg4 (magnésium du prélèvement 4) $(-0,61)$, argile $3(-0,60), M g 3(-0,58)$, la pluviométrie $(+0,57)$, argile $4(-0,55), M g 2(-0,48), \mathrm{K} 2(-0,47)$, Is $2(-0,44)$, K4 $(-0,44)$, l'indice de drainage $(+0,43)$.

Plusieurs régressions progressives ont été effectuées, pour "expliquer » la production à l'aide de différents groupes de variables. Les plus intéressantes fournissent les résultats suivants $\left({ }^{2}\right)$ :

a) Pour l'ensemble des placettes (94):

- en introduisant l'ensemble des variables retenues pour ce $2^{\mathrm{e}}$ stade, le coefficient de corrélation multiple (c.c.m.) au 5 e palier est de 0,81; 65 p. 100 de la variation de production sont expliqués à l'aide de :

- Mg4 + Pluviométrie - argile 3 - is $2+$ indice de drainage $\left(^{1}\right)$.

En fait à ce palier la pluviométrie n'est plus significative. En la supprimant, le c.c.m. est encore de 0,80.

- en n'introduisant pas Mg ni K, le c.c.m. au 3e palier est de 0,79; 62 p. 100 de la variation sont «expliqués» par :

$$
\text { - argile } 3 \text { - Is } 2+\text { indice de drainage }
$$

(1) - Mg4 signifie que le coefficient par lequel on multiplie Mg4 dans l'équation est négatif ; et ainsi de suite.

${ }^{2}$ ) Nous arrêterons toutes les régressions progressives au dernier palier pour lequel l'introduction de la nouvelle variable est significative, ou même avant si le coefficient de corrélation multiple n'est guère amélioré ensuite. 
- en supprimant les variables fluctuantes (nappe et pluviométrie) par rapport à la régression précédente; 58 p. 100 de la variation (c.c.m. $=0,76$ ) sont encore expliqués, par :

$$
\text { - Is } 2 \text { - argile } 4 \text { - argile } 2 \text { - argile } 3 .
$$

b) En supprimant les placettes à «zone saturée d'été » (quatre-vingt placettes demeurent), la production est «expliquée » par les groupes de variables suivantes :

$*+$ indice drainage - Is $2-$ argile $3-$ Mg4 (c.c.m. $=0,86$ )

$*$ - Is $2+$ indice de drainage - argile 3 - argile 4 (c.c. $=0,86$ )

$*$ - Is 2 - argile 4 - argile 2 - argile 3 (c.c.m. $=0,80$ ).

\subsection{Commentaires.}

- Les c.c.m. des équations retenues sont élevés; des chiffres semblables ont rarement été atteints au cours des précédentes enquêtes station-production.

- L'introduction des variables chimiques n'améliore guère ou même pas du tout les c.c.m. des équations ne comportant que des facteurs physiques. Nous ne retiendrons donc finalement que ces dernières, d'autant plus qu'il est probable que la teneur en $\mathrm{Mg}^{++}$ne fasse que traduire le pourcentage d'argile de l'horizon considéré. Nous conservons donc deux équations pour chacun des deux cas $a$ ) et $b$ ), la première (comprenant l'indice de drainage) intéressante sur le plan explicatif, la seconde (sans cet indice) valable pour la prévision de la production de peuplements non encore étudiés. On peut les schématiser en disant que la production est d'autant meilleure que la nappe temporaire est moins superficielle à une date de référence, que la texture du sol est moins argileuse dans ses 50 premiers $\mathrm{cm}$, et que sa structure est plus stable, entre -10 et $-20 \mathrm{~cm}$, c'est-à-dire, dans la majorité des cas, dans l'horizon minéral situé directement sous l'horizon $A_{1}$.

- La présence d'une "zone saturée d'été », qui apporte la présomption d'une meilleure alimentation en eau au cours des périodes de sécheresse, affaiblit le c.c.m. des équations «explicatives» de la production : ce phénomène contrebalance en partie l'effet défavorable de la nappe temporcire et des propriétés physiques du sol.

- II n'est pas facile d'expliquer parfaitement la production à l'aide des équations retenues. D'une part, nous ne sommes pas autorisés, en l'absence d'expérimentations destinés à confirmer les hypothèses émises, à parler de causalité : la présence de chaque facteur dans les équations retenues peut ne traduire que l'influence d'une autre variable, non prise en compte. Aussi, lorsque nous parlerons en termes de causalité ne faudra-t-il y voir que des hypothèses. D'autre part, même les trois variables mises en évidence (indice de drainage, teneur en argile, instabilité structurale) sont interdépendantes : on ne peut savoir quel est le facteur qui agit réellement le plus sur la production. Cependant, les équations retenues indiquent en principe que chacun des trois facteurs exerce une certaine action propre sur la production (leur coefficient de corrélation partielle avec la production est significatif) : par exemple l'indice de drainage est lié à la production, même à propriétés physiques du sol constantes. Pour l'argile, par contre, il pourrait y avoir un certain doute ; en effet, si la production est liée à la teneur en argile à indice de drainage identique, nous avons établi précédemment (voir le chapitre "Méthodes ») que, pour un même indice, l'engorgement (dans des horizons relativement superficiels) était plus long, au cours d'une année, quand 
l'argile apparaissait plus superficiellement. Donc une partie de la liaison argileproduction est probablement due à l'action de la texture sur la durée de la nappe à indice de drainage constant. Mais, en raison des coefficients de corrélations partielles élevés entre les teneurs en argile de différents horizons ef la production, on peut penser que la texture exerce sur la production une action indépendante de celle pratiquée sur la durée de la nappe.

- A titre indicatif, voici une des équations permettant de prévoir avec une certaine incertitude (écart-type résiduel $=1,96 \mathrm{~m}$ ) la hauteur dominante à 50 ans à l'aide des seules propriétés physiques du sol, pour des peuplements sans zone saturée d'été :

$$
\begin{gathered}
33,82-0,071 \times \text { argile } 2-2,63 \times \text { Is } 2-0,047 \times \text { argile } 3-0,072 \times \text { argile } 4 \\
=\text { HD } 50(\text { en m). }
\end{gathered}
$$

\section{2. - Examen des cas réels après regroupements}

De l'étude statistique se sont dégagés les facteurs dont dépend, semble-t-il, la production de l'Epicéa. Mais les équations « expliquant» cette production sont difficiles à interpréter sur un plan pratique : elles ne montrent pas les différents cas réels.

Aussi avons-nous groupé les placettes selon leur texture, propriété du sol apparemment la plus importante. Il nous fallait retenir, dans un but de simplification, une seule variable, et non les trois que l'étude statistique avait dégagées (teneur en argile des prélèvements 2,3 et 4 ) : nous avons choisi, après différents essais, la profondeur d'apparition de la texture "argileuse lourde », c'est-à-dire une teneur d'au moins 45 p. 100 d'argile, et créé empiriquement quatre groupes de placettes selon que cette valeur apparaissait à moins de $10 \mathrm{~cm}$ (groupe 1), entre 10 et $32 \mathrm{~cm}$ (groupe 2), entre 32 et $50 \mathrm{~cm}$ (groupe 3) ou à plus de $50 \mathrm{~cm}$ de profondeur (groupe 4). La plupart de nos stations peuvent se placer dans le cadre du Catalogue des stations du plateau lorrain (Brethes, 1976) ; voici, pour elles, la correspondance approximative :

- groupe de texture 1 : groupe de stations no 200

- groupe de texture 2 : stations nos 310 et 320

- groupe de texture 3 : stations $n^{0} 330$

- groupe de texture 4: groupe de stations $n^{\text {os }} 340$ et 400 .

Nous avons par ailleurs défini trois catégories de production, dont les limites sont les suivantes : en hauteur dominante à 50 ans (HD 50): $\geqslant 28,1 \mathrm{~m}$ (catégorie 1), de 25,0 à 28,0 m (catégorie 2) et $\leqslant 24,9 \mathrm{~m}$ (catégorie 3). Avec une petite distorsion, la catégorie 1 correspond approximativement à la classe 1 et au-dessus, la catégorie 2 à la classe 2 et la catégorie 3 aux classes 3 à 6 de la table de production de l'Epicéa pour le N.E. de la France (Decourt, 1972).

L'indice de drainage représente la nappe temporaire. Nous examinerons cependant ensuite si les niveaux de cette nappe à d'autres dates que celle choisie pour l'indice de drainage sont susceptibles d'améliorer l'interprétation.

\subsection{Examen des différents groupes de texture.}

Nous avons représenté (fig. 1) pour chaque groupe de texture la catégorie de production et la position des placettes en fonction de l'indice de drainage et de ls du 
2 e prélèvement (Is 2), exception faite de celles présentant une zone saturée d'été ; en effet, la présence de cette caractéristique modifie la production ; nous examinerons plus loin son influence réelle.

La droite tracée sur les trois premiers graphiques de cette figure sépare, de façon presque parfaite, les placettes des catégories de production 2 et 3 . Sur le 4 e graphique (4e groupe de texture), vingt des vingt-sept placettes représentées au-dessus de la droite appartiennent à la catégorie de production 1 ; les sept autres n'atteignent que la $2^{\mathrm{e}}$ catégorie de production ; en réalité, il se trouve que, sur ces sept placettes, deux présentent à profondeur moyenne dans leur sol une couche quasi continue de cailloux ; deux autres ont une texture en fait intermédiaire entre les groupes 3 et 4 (presque 45 p. 100 d'argile de -40 à $-50 \mathrm{~cm}$ ), et enfin la courbe de croissance de deux autres placettes montre un « démarrage » initial anormalement mauvais.

Nous avons effectué le même examen en utilisant le niveau d'affleurement de la nappe à d'autres dates que le 16.2.1971 qui avait été retenu comme indice de drainage : dates auxquelles les nappes sont en général plus profondes $(24.1 .1974)$ ou plus superficielles (11.2.1974).

Nous avons pu remarquer d'abord que le classement de l'ensemble des placettes quant au niveau de nappe est assez voisin aux trois dates. Quant à l'interprétation de la production à l'aide de chacune de ces deux nouvelles dates, elle n'apporte rien de plus, au contraire, que celle effectuée en utilisant l'indice de drainage. On peut en conclure que le niveau de nappe au 16.2.71 a été choisi à bon escient comme indice de drainage.

\subsection{Synthèse : influence respective des différents facteurs sur la production.}

Nous avons constaté, en conclusion de l'étude statistique, qu'il était très plausible que les facteurs qui s'en étaient dégagés (texture, indice de drainage, Is 2 , et aussi "zone saturée d'été ») soient ceux dont dépend réellement (liaisons de causalité) la production. L'examen des différents groupes de texture vient de nous permettre d'illustrer les interactions entre ces facteurs. Nous allons maintenant résumer le rôle de chacun d'eux, ainsi que celui, éventuel, d'autres variables dont l'influence sur la production est également possible.

1) «Zone saturée d'été ».

Quelle que valeur que prennent les autres variables, aucune placette n'a une mauvaise production (catégorie 3 ) en présence de cette caractéristique.

Dans les groupes de texture 1 à 3 , elle fait gagner une catégorie de production, parfois deux. Elle semble moins efficace pour les placettes du groupe 4 : deux placettes sur quatre conservent une production 2, mais rappelons que cette dénomination «zone saturée d'été » englobe des situations réelles fort diverses.

Les données qui suivent ne sont valables qu'en l'absence de cette «zone ».

2) La texture.

Elle agit, bien entendu, sur les niveaux d'affleurement de la nappe temporaire ; ainsi le nombre de placettes à indice de drainage inférieur à 20 est le suivant : 10 sur 15 dans le groupe 1, 4 sur 15 dans le groupe 2, 4 sur 20 dans le groupe 3 et 2 sur 40 dans le groupe 4. Mais il s'agit ici d'examiner l'action de la texture sur la production 
indépendamment de son influence sur l'indice de drainage. Rappelons, cependant, qu'une part de sa liaison avec la production est peut-être la conséquence du fait qu'à indice de drainage identique, la durée d'engorgement (dans des horizons relativement superficiels) au cours de l'année est d'autant plus longue que l'argile apparaît plus superficiellement (les différences ne sont cependant pas très importantes).

a) Lorsque l'indice de drainage est inférieur à 20 la production est à peu près toujours de catégorie 3, quel que soit le groupe de texture. Mais en valeur réelle, la production est presque toujours plus élevée dans les groupes 3 et 4 que dans les groupes 1 et 2.

b) Lorsque cet indice est égal ou supérieur à 20, toutes les autres conditions étant égales, la production dépend nettement de la texture : ainsi il y a un grand nombre de placettes à production 1 dans le groupe 4, aucune dans les trois autres groupes, du moins en l'absence de «zone saturée d'été ». D'autre part à indice de drainage comparable, la valeur critique de ls 2 (c'est-à-dire celle au-delà de laquelle la production passe à la catégorie suivante) est d'autant plus élevée que l'on passe du groupe 1 au groupe 4.

3) L'indice de drainage.

a) Lorsqu'il est inférieur à 20, la production est presque toujours de catégorie 3, indépendamment de la texture et de ls 2 .

b) Si les autres conditions sont favorables, une nappe temporaire peu marquée (indice supérieur à 25 ou 30) n'empêche pas d'atteindre de très bonnes productions (classe 1 de l'Epicéa dans le Nord-Est).

4) Is 2 (indice d'instabilité structurale du prélèvement 2, c'est-à-dire de -12 à-20 cm)

Is 2 semble n'avoir aucun rôle marquant lorsque l'indice de drainage est inférieur à 20 .

A indice supérieur à 20 : dans un même groupe de texture et pour un indice de drainage comparable, la catégorie de production dépend de la valeur is 2 (selon cette valeur catégorie 2 ou 3 dans les groupes de texture 1 à 3 , catégorie 1 ou 2 en général dans le groupe 4).

5) Le potentiel d'oxydo-réduction.

Mesurable uniquement en présence d'une nappe ou pour le moins en sol très humide, il est lié à la teneur du milieu en oxygène ou au contraire en composés réducteurs. Il est d'autant plus favorable aux racines que sa valeur est plus élevée.

Nous avons mesuré les potentiels redox de quatorze placettes réparties dans les quatre groupes de texture, mais toutes à nappe souvent superficielle, à plusieurs reprises au cours d'un hiver et d'un printemps (1975) sous des températures ambiantes variées. Les données recueillies sont surtout intéressantes lorsque la nappe est proche de la surface, c'est-à-dire quand elle affecte la zone principale d'enracinement.

- Potentiel redox relevé dans le sol (à -5 et $-9 \mathrm{~cm}$ ) : à une date donnée, il augmente, dans l'ensemble, du groupe 1 au groupe 4, c'est-à-dire de l'argile la plus superficielle à l'argile la plus profonde. II n'est pas rare cependant qu'il soit à peu près 
identique pour les placettes des groupes 1 et 2 , ainsi que pour celles des groupes 3 et 4 . Ainsi le 29 janvier, Eh varie de +40 à +105 mv pour les deux premiers groupes, de +110 à +190 mv pour les deux derniers (valeurs obtenues à l'aide d'une électrode de référence au calomel).

- Potentiel redox de la nappe (eau recueillie dans un bécher) : il est souvent assez différent de celui mesuré à la même profondeur dans le sol engorgé. II est cependant lié également à la texture du sol : ainsi le 29 janvier Eh varie de +85 à $+145 \mathrm{mv}$ dans les nappes des placettes des groupes 1 et 2 , de +145 à $+210 \mathrm{mv}$ dans celles des placettes des groupes 3 et 4 . On a même relevé des valeurs négatives dans les nappes des placettes des deux premiers groupes au cours du printemps (valeur la plus basse : $-130 \mathrm{mv}$, le 22 avril pour la placette 72 ).

6) Autres variables.

- L'humidité du sol en période de sécheresse : on peut penser à cette variable, car on sait que la difficulté d'alimentation en eau à certains moments de l'été est une des causes probables du caractère néfaste des pseudogleys vis-à-vis du peuplement.

Nous n'avons en fait pu dégager aucune liaison nette entre cette valeur, quel que soit le niveau de la mesure, ef la production : cela n'est pas étonnant, car les réserves en eau disponibles sont avant tout liées au volume prospecté par les racines.

- La pluviosité : il est troublant de constater que sur les quinze placettes où la pluviosité moyenne annuelle est inférieure à $800 \mathrm{~mm}$, douze ont une production 3 malgré un indice de drainage souvent supérieur à 20. Mais ls 2 a presque toujours une valeur élevée (pour le groupe de texture correspondant) : cette liaison, probablement aléatoire, entre une faible pluviosité et ls 2 élevé explique que la pluviosité n'apparaisse pas dans les régressions progressives les plus intéressantes. Si les précipitations peuvent être évidemment défavorables, car à l'origine des nappes temporaires, à indice de drainage identique la sécheresse d'été est certainement un facteur très néfaste en sol hydromorphe du fait d'un mauvais enracinement.

- Enfin, nous avons déjà signalé qu'une couche à très forte teneur en cailloux limitait la production (cas de deux placettes à production 2 au lieu de 1). Par ailleurs, la tourbe drainée (placette 31) constitue un bon substrat (HD $50=28,1 \mathrm{~m}$, production 1).

\section{3. - La nutrition}

\section{1. - Niveau général de nutrition. L'azote et sa variation d'une année sur l'autre}

Les coefficients de corrélation entre les teneurs en un même élément en 1969 et 1970 sont les suivants :

$$
\mathrm{N}: 0,18 ; \mathrm{P}: 0,81 ; \mathrm{K}: 0,71 ; \mathrm{Ca}: 0,60 ; \mathrm{Mg}: 0,58 ; \mathrm{Mn}: 0,71 \text {. }
$$

La liaison est donc pratiquement nulle pour $\mathrm{N}$, bonne pour les autres éléments. La nutrition azotée paraît donc dépendre énormément des conditions climatiques de l'année.

La nutrition en $\mathrm{P}, \mathrm{K}, \mathrm{Ca}, \mathrm{Mg}, \mathrm{Mn}$ est correcte dans l'ensemble. Les concentrations ne sont jamais inférieures au seuil de carence, elles dépassent même souvent l'opti- 
mum. Cela n'a d'ailleurs rien d'étonnant sur des sols à humus actifs et dont la teneur en argile est suffisante, voire même souvent excessive.

Quant à l'azote, examinons sa concentration foliaire par rapport aux chiffres, souvent cités, de seuil de carences $(1,20$ à 1,25$)$ et d'optimum $(1,50$ p. 100) :

- placettes à concentration foliaire en $N$ inférieure ou égale à 1,20 p. 100 :

1969 : huit placettes (9 p. 100)

1970 : vingt-quatre placettes (27 p. 100)

- placettes à concentration foliaire en $N$ inférieure ou égale à 1,25 p. 100 :

1969 : douze placettes (13 p. 100)

1970 : trente-sept placettes (41 p. 100)

- placettes à concentration foliaire en $N$ supérieure ou égale à 1,50 p. 100 :

1969 : dix-sept placettes (19 p. 100)

1970 : deux placettes (2 p. 100).

II y a donc un décalage général vers les faibles valeurs en 1970 par rapport à 1969.

II en découle qu'on ne peut pas se contenter d'une seule analyse d'azote pour juger des conditions de nutrition d'un peuplement situé sur sol hydromorphe : il est nécessaire de renouveler au moins une fois l'opération à l'automne d'une année à caractéristiques climatiques nettement différentes.

L'année 1969 fut nettement plus sèche que 1970, en toutes saisons. Par rapport aux valeurs moyennes, l'hiver et le printemps 1970 ont été particulièrement humides. La nutrition azotée de l'Epicéa paraît donc diminuée, en sol hydromorphe, par une forte pluviosité, par l'intermédiaire de la nappe temporaire vraisemblablement.

Une remarque concernant la valeur de l'optimum foliaire en azote s'impose à la vue des résultats analytiques : la placette 34 a des teneurs en azote de 1,31 p. 100 en 1969 et 1,35 p. 100 en 1970 ; or sa production est remarquable, probablement parmi les toutes meilleures de Lorraine ; des remarques similaires peuvent être faites pour d'autres placettes; le chiffre de 1,50 p. 100 pour la teneur optimum semble donc nettement trop élevé, au moins dans certains cas ; ou alors des valeurs d'azote inférieures à l'optimum n'affectent que très peu la croissance au moins jusqu'aux environs de 1,31 p. 100 . Bien sûr, nous comparons des teneurs annuelles en azote à une production moyenne et non à l'accroissement courant ; mais le fait qu'un peuplement atteigne des productions comparables à celles de la placette 34 exclut pratiquement l'éventualité d'accroissements faibles certaines années.

Il faut cependant signaler que la méthode analytique utilisée pour l'azote (attaque à $\mathrm{SO}_{4} \mathrm{H}_{2}+\mathrm{H}_{2} \mathrm{O}_{2}$ en l'absence de sélénium) n'est pas parfaite et peut aboutir dans certains cas à des résultats quelque peu inférieurs (5 p. 100) à la réalité.

Enfin, remarquons que si la teneur en $\mathrm{N}$ des feuilles de l'année obtenue sur des prélèvements d'automne synthétise en principe la nutrition azotée de l'ensemble de la saison de végétation, cela n'exclut pas l'éventualité d'une mauvaise nutrition au cours du printemps, qui serait « rattrapée » par des conditions favorables au cours de l'été : des carences passagères peuvent ainsi passer inaperçues. 


\section{2. - Liaisons nutrition-sol}

3.21. L'azote foliaire.

Il apparaît au premier abord une liaison très nette entre cet azote et la texture du sol. Le tableau 2 fait apparaître la liaison entre la nutrition azotée et les groupes de texture définis précédemment.

TABLEAU 2

Nutrition azotée et groupes de texture

Nitrogen nutrition and texture groups

\begin{tabular}{|c|c|c|c|c|c|c|c|c|c|c|c|c|}
\hline & \multicolumn{4}{|c|}{$N \leqslant 120$ p. 100} & \multicolumn{4}{|c|}{$N \leqslant 125$ p. 100} & \multicolumn{4}{|c|}{$N \geqslant 150$ p. 100} \\
\hline & \multicolumn{2}{|c|}{1969} & \multicolumn{2}{|c|}{1970} & \multicolumn{2}{|c|}{1969} & \multicolumn{2}{|c|}{1970} & \multicolumn{2}{|c|}{1969} & \multicolumn{2}{|c|}{1970} \\
\hline & Nbre & p. 100 & Nbre & p. 100 & Nbre & p. 100 & Nbre & p. 100 & Nbre & p. 100 & Nbre & p. 100 \\
\hline $\begin{array}{l}\text { Groupe } 1 \\
\text { (15 placettes) }\end{array}$ & 3 & 20 & 10 & 67 & 4 & 27 & 12 & 80 & 4 & 27 & 0 & 0 \\
\hline $\begin{array}{l}\text { Groupe } 2 \\
\text { (15 placettes) } \ldots \ldots \ldots\end{array}$ & 3 & 20 & 6 & 40 & 4 & 27 & 7 & 47 & 2 & 13 & 0 & 0 \\
\hline $\begin{array}{l}\text { Groupe } 3 \\
\text { (20 placettes) }\end{array}$ & 2 & 10 & 4 & 20 & 3 & 15 & 8 & 40 & 4 & 20 & 1 & 5 \\
\hline $\begin{array}{l}\text { Groupe } 4 \\
\text { (40 placettes). }\end{array}$ & 0 & 0 & 4 & 10 & 1 & 2,5 & 10 & 25 & 7 & 18 & 1 & 2,5 \\
\hline
\end{tabular}

Cette liaison est très nette pour les basses valeurs d'azote. Il y a en 1970, d'autant moins de placettes carencées que l'on passe du groupe 1 au groupe 4. Ce résultat apparaît également en 1969, mis à part le fait que les deux premiers groupes ont alors des valeurs identiques. Le nombre de placettes carencées est bien plus élevé dans chaque groupe en 1970 qu'en 1969. Par contre, il n'apparaît pas de liaison entre les fortes valeurs en azote et le groupe de texture.

On peut penser que la texture intervient sur la nutrition azotée au moins en grande partie par l'intermédiaire de son influence sur le niveau et les fluctuations de la nappe temporaire : le fait que beaucoup plus de placettes sont carencées en 1970, année humide, qu'en 1969 renforce cette hypothèse.

En 1970, il existe en effet une liaison très nette entre teneur foliaire en azote (N 70) et indice de drainage. Ainsi parmi les vingt-et-une placettes à indice inférieur à 20 , vingt ont $N 70<1,31$ p. 100 . D'autre part, douze des treize placettes à indice inférieur à 15 ont $N 70<1,24$ p. 100 . Enfin, parmi les vingt-quatre placettes à $N 70<$ 1,20 p. 100 , vingt ont un indice de drainage $\leqslant 30$.

En 1969, année relativement sèche, la nappe temporaire ne limite sans doute la teneur foliaire en azote ( $N$ 69) que dans un plus petit nombre de placettes. Un faible indice de drainage n'est de loin plus la seule condition nécessaire pour faire apparaître une carence azotée : d'une part une texture favorable (groupes 3 et 4) permet souvent d'éviter cette carence, et par ailleurs la présence d'une «zone saturée d'été » se tra- 
duit toujours par une nutrition azotée correcte. Parmi les placettes les moins riches en azote $(N 69 \leqslant 1,30$ p. 100$)$ une dizaine ont ainsi un indice de drainage très faible, donc sans doute limitant ; les autres ont presque toutes une valeur très élevée de Is 2 (indice d'instabilité structurale de $-12 \mathrm{à}-20 \mathrm{~cm}$ ); nous savons que cela se traduit par un enracinement relativement superficiel, donc probablement un déficit hydrique au cours des étés assez secs, comme en 1969.

\subsection{Le magnésium.}

C'est le seul élément foliaire qui, en dehors de l'azote, semble lié quelque peu au caractère plus ou moins hydromorphe des placettes.

La teneur des aiguilles en Mg est en moyenne d'autant plus élevée que l'argile est plus superficielle. Cela explique-t-il que la liaison entre la teneur foliaire en $\mathrm{Mg}$ et l'indice de drainage soit surtout net pour des placettes du groupe 4 ? Toujours est-il que dans ce groupe une forte teneur en $\mathrm{Mg}$ est très liée à un faible indice de drainage. La liaison est presque aussi bonne pour les placettes du groupe 3, beaucoup moins pour celles du groupe 2 ; elle n'existe pas dans le groupe 1.

\section{3. - Liaisons nutrition-production}

\subsection{En 1969.}

Le coefficient de corrélation totale le plus élevé (en valeur absolue) avec l'indice de production, parmi l'ensemble des éléments minéraux contenus dans les aiguilles, est celui du magnésium : - 0,45 (très significatif) ; la teneur en $\mathrm{Mg}$ 《explique » donc 21 p. 100 de la variation totale de la production. En seconde position apparaît l'azote avec un coefficient de $+0,36$ (très significatif également). D'ailleurs la hauteur dominante est liée négativement à tous les éléments majeurs sauf l'azote.

L'analyse par régressions progressives est intéressante jusqu'au $4^{\mathrm{e}}$ palier (variables introduites successivement : $\mathrm{Mg}, \mathrm{N}, \mathrm{Mn}, \mathrm{Ca}$, avec un coefficient positif pour l'azote, négatif pour les autres éléments) car au-delà l'introduction des nouvelles variables n'est plus significative. L'équation correspondante a un coefficient de corrélation multiple de 0,63 , elle "explique » 40 p. 100 de la variation de la production.

L'analyse des composantes principales et celle des correspondances continues (dans laquelle on considère la variation relative des différentes teneurs foliaires, et non leur valeur absolve comme dans le cas des composantes principales) ne permettent qu'assez peu d'améliorer la compréhension des liaisons entre la nutrition et la production. La seconde fait cependant apparaître une certaine liaison entre basse production d'une part, teneurs faibles en $\mathrm{N}$ et élevées en $\mathrm{P}, \mathrm{K}, \mathrm{Ca}, \mathrm{Mg}$ d'autre part.

En fait, l'interprétation de ces analyses est délicate, et on ne peut en tirer que des enseignements indicatifs et qualitatifs. Elles permettent surtout de faire apparaître des liaisons linéaires. Ce n'est pas forcément le cas dans le problème qui nous occupe. Les notions bien établies de seuils de carence foliaires et de facteurs limitants suggèrent la possibilité de liaisons entre production et nutrition qui n'existeraient que pour certaines gammes de concentration foliaire. Pour détecter ces liaisons non linéaires, C. Millier (1973) a effectué l'analyse des correspondantes discrètes, pour laquelle chaque facteur (les différents éléments nutritifs dans les feuilles ainsi que l'indice de 
production HD 50) a été réparti en quatre classes, chacune intervenant dans l'analyse en tant que variable. Notons que cette étude de $C$. Millier était la première où l'analyse des correspondances discrètes était appliquée à un problème forestier, en vue de la détection de liaisons non linéaires. L'analyse du 1 er vecteur propre (1er facteur) (fig. 2) montre, en reliant les quatre points relatifs à chaque caractère (par exemple $\mathrm{N} 1, \mathrm{~N} 2, \mathrm{~N} 3, \mathrm{~N}$ 4) que $\mathrm{P}, \mathrm{K}, \mathrm{Ca}, \mathrm{HD} 50$ et surtout $\mathrm{Mg}$ ont une trajectoire qui croît selon un ajustement qui pourrait être linéaire, alors que celle de $\mathrm{N}$ est asymptotique :

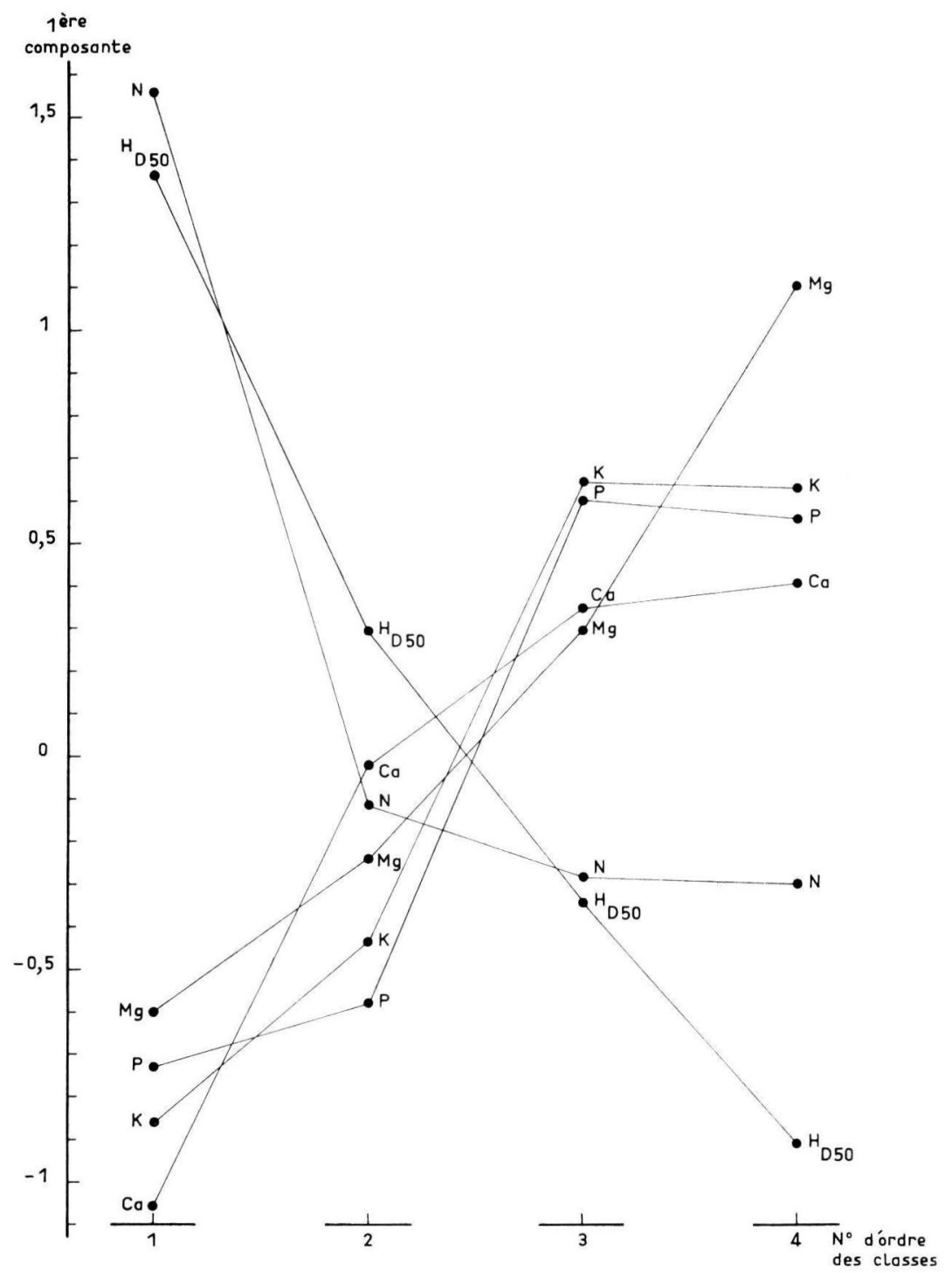

FIG. 2. - Analyse des correspondances discrètes: $1^{\text {re }}$ composante relative aux différents caractères (C. Millier, 1973).

Correspondence analysis with splitting of quantitative variable : first componant relating to the various characters (C. Millier, 1973). 
les liaisons entre les premiers caractères sont sans doute à peu près linéaires, alors que $N$ est en liaison non linéaire avec les autres caractères et en particulier avec l'indice de production HD 50 : une faible teneur en $N$ dans les aiguilles (1 re classe de $\mathrm{N}$ ) est liée en général à une mauvaise production (classe regroupant les plus faibles valeurs de HD 50) (voir en effet la proximité de ces deux variables sur la figure 2). Au-delà de la 1 re classe, il n'y a plus de liaison entre $N$ ef production (HD 50 continue à descendre, alors que $N$ s'asymptotise sur cette figure 2).

Un examen de la teneur foliaire en azote dans les placettes confirme bien cette liaison : des valeurs inférieures à 1,23 p. 100 (qui est la limite entre les classes d'azote 1 et 2) correspondent, dans neuf cas sur dix, à des placettes à mauvaise production.

\subsection{En 1970.}

Rappelons que pour des éléments autres que $\mathrm{N}$, il y a une corrélation élevée entre les teneurs en 1969 et 1970 : les liaisons avec la production sont voisines de ce qu'elles étaient en 1969.

Ce n'est pas le cas de l'azote. Il existe une certaine liaison entre de faibles teneurs foliaires en azote et une faible production mais bien moins nette qu'en 1969 ; cela est dô en partie (mais pas uniquement) au fait que les placettes à "zone saturée d'été 》 n'ont jamais une production mauvaise alors que leur concentration foliaire en azote est souvent faible en 1970.

\section{4. - Synthèse}

Il est logique que les teneurs foliaires en $\mathrm{P}, \mathrm{K}, \mathrm{Ca}, \mathrm{Mg}$ soient en général élevées en sol à hydromorphie temporaire, et cela quelles que soient les conditions climatiques de l'année, car l'argile, à complexe absorbant important ef souvent saturée en bases tient un rôle important dans ces sols. Il est non mcins logique que ces teneurs soient liées négativement à la production car de l'argile, facteur défavorable à la croissance, dépend en partie l'engorgement, autre facteur nuisible. Le magnésium est, parmi ces éléments, le plus lié à la production, probablement parce que sa teneur est corrélée négativement avec l'indice de drainage dans certains groupes de texture.

L'azote, au contraire, prend souvent des valeurs très faibles dans les aiguilles, et ce phénomène est fortement lié au climat de l'année. Il semble provcqué par la nappe temporaire, particulièrement défavorable au cours des années humides, ou sinon, apparemment, par le dessèchement au cours des étés secs des zones enracinées, ce qui se traduit sans doute par une mauvaise minéralisation et surtout une faible absorption de $\mathrm{N}$ dans les sols où les racines ne peuvent se propager correctement en profondeur (mauvaise stabilité structurale); une alimentation par voie montante ("zone saturée d'été ») permet d'éviter ce deuxième phénomène. La liaison entre faible concentration foliaire en azote et basse production est logique.

\section{4. - Discussion et conclusion}

\section{1. - Discussion}

La production de l'Epicéa en sol hydromorphe apparaît très liée à trois facteurs édaphiques : l'argile, la stabilité structurale du sol, l'indice de drainage. Nous avons 
exposé les aspects quantitatifs de ces liaisons. Outre leur évidente interdépendance, chacune de ces variables a un rôle propre.

Même en l'absence d'expérimentation, il apparaît probable que cela constitue la cause première de la variation de production.

Ces facteurs, en effet, conditionnent fortement l'enracinement :

a) l'indice de drainage : la présence plus ou moins longue d'une nappe superficielle tend à provoquer l'asphyxie des racines, et diminue profondeur et densité de l'enracinement : nous l'avons constaté expérimentalement sur de jeunes plants d'Epicéa (Levy, 1971) et Delvaux (1968) l'a observé sur des adultes ;

b) une structure instable du sol diminue la densité d'enracinement de l'Epicéa adulte (Levy, 1968) ;

c) l'argile, indépendamment de son influence sur les fluctuations de la nappe affecte sans doute les racines par rapport à des textures moins fines : nous avons d'une part vu qu'en présence de nappe le potentiel redox des horizons $A_{1}$ était plus bas en cas de forte proportion d'argile dans la terre fine à faible profondeur ; d'autre part (étude citée ci-dessus), à profondeur et autres conditions identiques, l'enracinement est moins dense si la texture est plus argileuse, même en sol non hydromorphe.

En dehors de l'enracinement proprement dit, il est connu que les conditions d'aération du milieu influencent la mycorhization des racines: les mycorhises sont avides d'oxygène, elles ne se développent bien que dans les sols bien aérés (Rouquerol 1967, Dommergues et Mangenot 1970) ; les quelques observations que nous avons effectuées dans ce domaine le confirment. Nous avons en effet effectué un complage approximatif du nombre des mycorhizes par unité de longueur sur des racines longues de diamètre $\leqslant 2 \mathrm{~mm}$ prélevées dans l'horizon $A_{1}$ de vingt-deux placettes, sur deux ou trois arbres par placette, représentant tous les groupes de texture et intensités d'hydromorphie. Nous n'avons pas trouvé de résultat bien net à l'aide de la densité totale des mycorhizes, en dehors du fait qu'elle est en général plus élevée dans le groupe 4 de texture que dans les autres groupes. Par contre, nous avons trouvé des liaisons intéressantes en affectant à chaque arbre un indice, de 1 à 5 , représentant très approximativement le pourcentage de mycorhizes vivantes par rapport au nombre total de mycorhizes; on obtient ainsi, pour chaque placette étudiée, un indice $M$ de survie des mycorhizes (tabl. 1). $M$ augmente en général avec l'indice de drainage et, si l'on ne considère que des placettes à indice de drainage $=50$ (absence de nappe à la date de référence), Maugmente du groupe 2 au groupe 4 (le groupe 1 n'est pas représenté dans l'échantillon) ce qui correspond aussi à un accroissement de la production. On peut en conclure que ce qui détermine $M$ est en premier lieu la valeur de l'indice de drainage et en second lieu, c'est-à-dire en l'absence d'indice bas, la texture.

Cette forte diminution des surfaces de contact entre l'arbre et le sol (racines proprement dites et, semble-t-il, mycorhizes actives) se traduit selon toute vraisemblance par un déficit de l'alimentation en eau au cours des périodes de sécheresse.

Les trois facteurs édaphiques en cause influencent aussi l'alimentation azotée des arbres, nous l'avons vu, et cela peut aller jusqu'à la carence (ce phénomène est peutêtre en partie lié à la diminution de l'enracinement ; il est peut-être aussi en partie la 

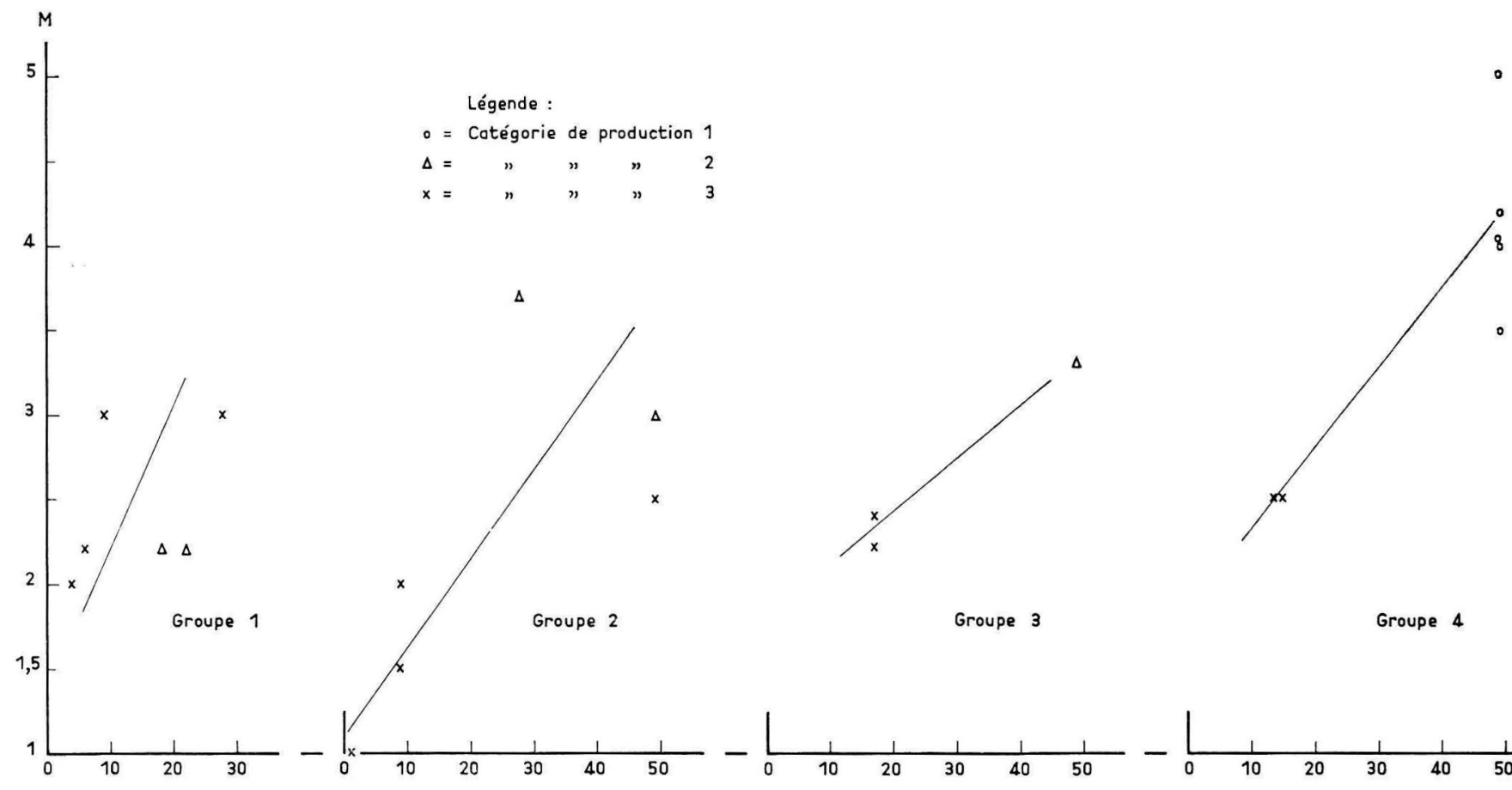

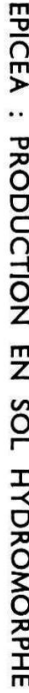

FIG. 3. - Variation de l'indice M (indice de survie des mycorhizes) en fonction de l'indice de drainage, par groupe de texture. Variation of « $M$ » index (mycorrhizae survival index) in connection with drainage index, by fexture group. 
conséquence de l'évolution des formes d'azote en sol engorgé : nous étudions ce problème par ailleurs).

Nous pouvons donc supposer en définitive que lorsque les trois facteurs édaphiques en cause prennent des valeurs défavorables, la production de l'Epicéa est affectée par l'intermédiaire d'un déficit d'alimentation en eau (la présence d'une zone profonde saturée en eau l'été permet en effet de restreindre cette perte de production) et d'une carence en azote (nous avons vu qu'une faible concentration de cet élément dans les aiguilles d'Epicéa correspondait presque toujours à une mauvaise production).

\section{2. - Conclusions pratiques}

Nous avons mis en évidence une diminution de production de l'Epicéa lorsque l'aération du milieu diminue, et en particulier quand l'hydromorphie augmente ; cela n'est pas surprenant, mais n'était à priori pas évident pour une espèce qui avait la réputation de bien supporter les sols hydromorphes.

La production reste toutefois intéressante dans la grande majorité des stations concernées. Elle ne devient vraiment faible (classes 4, 5 ou 6 de Decourt) que dans environ 10 p. 100 des placettes, presque toutes situées dans le groupe 2 de texture (argile apparaissant entre -10 et $-32 \mathrm{~cm}$, recouverte donc d'une mince couche limoneuse) : il s'agit très souvent de pélosols-pseudogleys.

Sur le plan pratique, notre étude peut fournir au reboiseur une idée de la production potentielle de l'Epicéa dans des stations situées dans la même gamme. Il aura ainsi un élément de décision pour le choix de l'essence à installer (pour des régions de climat différent, des organismes de développement pourraient, sur la base de nos résultats, effectuer une étude simplifiée des liaisons station-production).

Tout d'abord, des formules peuvent permettre de calculer très approximativement la production potentielle, par exemple celle citée à la fin du chapitre 2.12 (elle ne tient pas compte de l'indice de drainage, qui ne peut être exactement connu). Mais elles nécessitent des analyses de laboratoire.

Le praticien peut par ailleurs obtenir rapidement une certaine idée des potentialités de la station pour l'Epicéa :

- La texture du sol, seule, est déjà un indice intéressant. II est nécessaire de savoir la déterminer approximativement au toucher, en particulier de pouvoir reconnaître une texture argileuse lourde ( $\geqslant 45$ p. 100 d'argile).

Le tableau 3 mentionne la répartition approximative des placettes, en p. 100 , dans les différentes classes de production (table de production de Decourt) pour chaque groupe de texture ; c'est une indication pour le praticien. On peut ainsi constater, en l'absence de zone saturée d'été, que les classes de production 0 et 1 ne se rencontrent que dans le $4^{\mathrm{e}}$ groupe de texture, que dans les groupes 3 et 4 il n'y a pas de production plus faible que celle de la classe 3 , que les productions les plus faibles (classes 5 et 6 ) ne s'observent que dans le groupe 2, et que les moyennes approximatives de production sont les suivantes :

groupe 2 : entre classes 3 et 4

groupe 1 : classe 3

groupe 3 : entre classes 2 et 3

groupe 4 : entre classes 1 et 2 . 
TABLEAU 3

Répartition approximative des placettes par classes de production dans chaque groupe de texture

- pourcentage par rapport à l'ensemble des placettes du groupe,

- caractéristiques de la nappe temporaire ef de la structure du sol.

- $\mathrm{N}+\quad$ : nappe superficielle (à certaines dates),

- $N$ - $\quad$ nappe jamais superficielle (et en général peu épaisse),

- $\mathrm{N}+$ - : nappe de profondeur et d'épaisseur moyennes (groupe 4),

- $\mathrm{S}+$ : structure favorable,

- S - : structure non favorable.

Approximate distribution of the plots by yield class in each texture group :

- percentage of the whole plots of the group,

- caracteristics of the temporary water table and of the soil structure.

- $\mathbf{N}+\quad$ : shallow water-table (at least during some periods),

- N - : no water table, or deep and generally thin water table,

- $N+-$ : water table of mean depth and thickness (group 4).

- $\mathrm{S}+$ : favourable structure,

- $S-\quad$ : unfavourable structure).

\begin{tabular}{|c|c|c|c|c|c|c|c|c|}
\hline \multirow{2}{*}{$\begin{array}{l}\text { Groupe de } \\
\text { texture et } \\
\text { niveau } \\
\text { d'apparition } \\
\text { de la texture } \\
\text { argileuse } \\
\text { lourde, en cm }\end{array}$} & \multicolumn{7}{|c|}{ Classe de production (en l'absence d'une zone saturée d'été) } & \multirow{2}{*}{$\begin{array}{l}\text { Classe de } \\
\text { production } \\
\text { en présence } \\
\text { d'une zone } \\
\text { saturée d'été }\end{array}$} \\
\hline & 0 & 1 & 2 & 3 & 4 & 5 & 6 & \\
\hline $\begin{array}{c}\text { Groupe } 1 \\
(0-10)\end{array}$ & & & $\begin{array}{l}30 \text { p. } 100 \\
N- \\
S+\end{array}$ & $\begin{array}{l}60 \text { p. } 100 \\
N+ \\
S+\end{array}$ & $\begin{array}{l}10 \text { p. } 100 \\
N+ \\
S-\end{array}$ & & & $\begin{array}{c}\text { en général } \\
\text { classe } 2\end{array}$ \\
\hline $\begin{array}{c}\text { Groupe } 2 \\
(10-32)\end{array}$ & & & $\begin{array}{l}25 \text { p. } 100 \\
\mathrm{~N}- \\
\mathrm{S}+-\end{array}$ & $\begin{array}{l}25 \text { p. } 100 \\
\mathrm{~N}- \\
\mathrm{S}-\end{array}$ & $\begin{array}{l}35 \text { p. } 100 \\
s-\end{array}$ & $\begin{array}{l}\overline{7,5 \text { p. } 100} \\
s-\end{array}$ & $\begin{array}{l}\overline{7,5 \text { p. } 100} \\
N- \\
S-\end{array}$ & néant \\
\hline $\begin{array}{c}\text { Groupe } 3 \\
(32-50)\end{array}$ & & & $\begin{array}{l}60 \text { p. } 100 \\
N- \\
S+\end{array}$ & $\begin{array}{l}\overline{40 \text { p. } 100} \\
S-\end{array}$ & & & & 1 ou 2 \\
\hline $\begin{array}{c}\text { Groupe } 4 \\
(>50)\end{array}$ & $\begin{array}{l}10 \text { p. } 100 \\
N- \\
S+\end{array}$ & $\begin{array}{l}40 \text { p. } 100 \\
\mathrm{~N}- \\
\mathrm{S}+\end{array}$ & $\begin{array}{l}40 \text { p. } 100 \\
N+- \\
\text { ou } \mathrm{S}- \\
\text { ou nombreux } \\
\text { cailloux }\end{array}$ & $\begin{array}{l}10 \text { p. } 100 \\
N+\end{array}$ & & & & 1 ou 2 \\
\hline
\end{tabular}

- Cette prévision de la production à partir de la texture seule peut en principe être améliorée si l'on fait intervenir la stabilité structurale du sol et l'indice de drainage.

- Si l'on veut éviter une analyse de laboratoire, on peut souvent obtenir une certaine idée de la stabilité structurale simplement par examen du profil. A texture identique, la structure pourra être considérée comme favorable dans deux cas (ces données résultent d'observations effectuées dans les placettes) : présence soit d'un horizon organique $\left(A_{1}\right.$ ou $A_{p}$ ) assez épais (par exemple $\geqslant 15 \mathrm{~cm}$ ) et bien individualisé, soit, sous $A_{1}$ et jusqu'à au moins -25 ou $-30 \mathrm{~cm}$, d'un horizon nettement brunifié : teinte brune différente de celle de la roche-mère et structure en petits polyèdres bien distincts les uns des autres et stables. On peut ainsi expliquer la meilleure production d'ensemble dans le groupe 1 de texture par rapport au groupe 2 par la présence fré- 
quente, dans le 1 er cas, d'un mull « pélosolique » (souvent caractéristique des pélosols) assez épais, intermédiaire, pour beaucoup de ses particularités, entre mull eutrophe et mull calcique.

- Quant à l'indice de drainage (nous avons choisi dans cette étude le niveau d'affleurement de la nappe le 16.2.1971), il est évidemment impossible à connaître dans une station non encore étudiée ; par ailleurs les caractéristiques d'une nappe sont différentes selon que l'on ait affaire à un peuplement de feuillus ou d'Epicéa (nappe plus profonde dans ce $2^{\mathrm{e}}$ cas : Levy 1969). Le praticien peut néanmoins classer très qualitativement les stations qui l'intéressent en observant la profondeur d'apparition et la netteté des traces d'hydromorphie ainsi que les niveaux relatifs d'affleurement des nappes au cours de l'hiver ; il lui sera en particulier possible de distinguer les stations où apparaît fréquemment une nappe très superficielle (qui correspondraient grossièrement aux placettes à indice de drainage $<20$ ) ; ce caractère de forte hydromorphie peut d'ailleurs être confirmé dans les peuplements d'Epicéa existants, par observation foliaire (à défaut d'analyse) au cours d'une année humide : il y apparaît alors en général une carence azotée (aiguilles petites et jaunâtres) ; cette carence peut toutefois se manifester dans des stations peu hydromorphes.

Le tableau 3 résume schématiquement, selon les critères qui viennent d'être définis, ce qui distingue les placettes d'un même groupe de texture mais de classes de production différentes.

Ce tableau (en dehors de la dernière colonne) concerne les placettes ne bénéficiant pas d'un apport d'eau profond (zone saturée d'été). La présence éventuelle d'une telle zone pourra être facilement détectée par le praticien par sondage du sol en profondeur au cours d'une période de sécheresse. Une zone saturée d'été permet en moyenne de gagner une classe de production, sauf dans le $4^{\mathrm{e}}$ groupe de texture, où elle ne semble pas avoir d'influence marquée.

Ce tableau permettra au praticien de prévoir approximativement la ou les classes de production correspondant à la station qui l'intéresse. En fait, il ne s'agira que d'une classe probable mais non certaine : c'est la conséquence de la schématisation assez poussée que nous avons volontairement adoptée dans ce tableau, ainsi d'ailleurs que du fait que certains facteurs de variation, tel le patrimoine génétique des arbres, nous ont totalement échappé.

Reçu pour publication en juillet 1977.

\section{Remerciements}

Nos remerciements s'adressent tout spécialement à C. Millier, Directeur de la Station de biométrie du C.N.R.F., pour les calculs et leur interprétation, en particulier ce qui concerne l'analyse des correspondances discrètes, et à D. G. Strullu, enseignant à la faculté des Sciences de Rennes, qui nous a fait bénéficier de son expérience sur les mycorhizes.

\section{Summary}

Nutrition and production of adult Norway Spruce on hydromorphic soils in Lorraine, in connection with site characteristics

Norway spruce is very much used in the East of France for afforesting hydromorphic soils. This site-nutrition-production study was done in Lorraine in a large diversity of sites. 
The production (from 4 to $22 \mathrm{~m}^{3} / \mathrm{ha} /$ year) is connected with the clay content of soil, with the stability of its structure, and with the drainage index. The multiple correlation coefficient is 0.86 for the sites without any deep permanent water table besides a shallow temporary one. When a permanent water table is present, the productivity of the site is, in average, one yield class higher.

Foliar analysis mostly showed a nitrogen deficiency, mainly during wet years and in the sites with a low drainage index.

Shallow water-table, bad structural stability and hight clay content reduce the soil volume prospecied by root system and therefore decrease water supply. Nitrogen and water deficiencies are probably the real cause of the production fall.

Soil profile and water table survey may give to the practitioner some idea of potential production of Spruce in the various hydromorphic sites.

\section{Références bibliographiques}

BRETHES A., 1976. Catalogue des stations forestières du plateau lorrain. O.N.F. et I.N.R.A., publication interne, $213 \mathrm{p}$.

DECOURT N., 1972. Tables de production provisoires pour l'Epicéa commun dans le N.E. de la France. Ann. Sci. forest., 29 (1) 49-63.

DECOURT N., LEVY G., 1974. - La production de l'Epicéa commun sur les sols hydromorphes lorrains : méthodes de mesure et valeurs. Ann. Sci. forest., 31 (2) 71-82.

DEI.VAUX J., 1968. Types de sol, enracinements et production. Observations concernant I'Epicéa, les Sapins et le Douglas. Bull. Soc. Roy. for. Belgique (1), 3-6.

DOMMERGUES Y., MANGENOT F., 1970. Ecologie microbienne du sol. Masson et Cie, Paris, $796 \mathrm{p}$.

GARBAYE ef al., 1970. Réflexions sur une méthode d'étude des relations entre facteurs écologiques et caractéristiques des peuplements. Ann. Sci. forest., 27 (3), 303-321.

LEVY G., 1968. Importance des propriétés du sol pour l'enracinement de Picea excelsa et de Pinus sylvestris. Ann. Sci. forest., 25 (3), 157.188.

LEVY G., 1969. Premiers résultats d'étude comparée de la nappe temporaire des pseudogleys sous résineux et sous feuillus. Ann. Sci. forest., 26 (1), 65-79.

LEVY G., 1971. Influence de l'engorgement du printemps et de la sécheresse d'été sur le comportement de jeunes plants d'Epicéa. Ann. Sci. forest., 28 (4), 403-423.

LEVY G., 1975. Nutrition de l'Epicéa commun en sols hydromorphes et lourds. Ref. Ecol. Biol. vol., 12 (1), 349-360.

MILLIER C., 1973. Une utilisation particulière de l'analyse factorielle des correspondances : détection de liaisons non linéaires entre variables. C.N.R.F., station de biométrie, publication interne, 5 p.

ROUQUEROL T., 1967. Physiologie et écologie des mycorhizes ectotrophes. Oecol. plant. (2), 85-124. 